\title{
Lapatinib sensitivities of two novel trastuzumab-resistant HER2 gene-amplified gastric cancer cell lines
}

\author{
Yukiko Oshima $\cdot$ Harunari Tanaka $\cdot$ \\ Hiroki Murakami $\cdot$ Yuichi Ito $\cdot$ Tomomi Furuya \\ Eisaku Kondo • Yasuhiro Kodera • Hayao Nakanishi
}

Received: 16 April 2013/Accepted: 28 July 2013/Published online: 15 August 2013

(c) The International Gastric Cancer Association and The Japanese Gastric Cancer Association 2013

\begin{abstract}
Background Trastuzumab (Tmab) resistance is a major clinical problem to be resolved in patients with HER2positive gastric cancers. However, in contrast to the situation for HER2-positive breast cancer lines, the Tmabresistant gastric cancer preclinical models that are needed to develop a new therapy to overcome this problem are not yet available.

Methods We developed three new cell lines from HER2 gene-amplified gastric cancer cell lines (GLM-1, GLM-4, NCI N-87) by a new in vivo selection method consisting of the repeated culture of small residual peritoneal metastasis but not subcutaneous tumor after Tmab treatment. We then evaluated the anti-tumor efficacy of lapatinib for these Tmab-resistant cells.

Results We successfully isolated two Tmab-resistant cell lines (GLM1-HerR2(3), GLM4-HerR2) among the three tested cell lines. These resistant cells differed from the parental cells in their flat morphology and rapid growth in vitro, but HER2, P95HER2 expression, and Tmab binding were essentially the same for the parental and resistant cells. MUC4 expression was up- or downregulated depending on the cell line. These resistant cells were still sensitive to
\end{abstract}

Y. Oshima $\cdot$ H. Murakami $\cdot$ Y. Kodera

Department of Gastroenterological Surgery, Nagoya University

Graduate School of Medicine, Nagoya, Japan

H. Tanaka $\cdot$ T. Furuya $\cdot$ E. Kondo $\cdot$ H. Nakanishi $(\bowtie)$ Division of Oncological Pathology, Aichi Cancer Center Research Institute, 1-1 Kanokoden, Chikusa-ku, Nagoya, Japan e-mail: hnakanis@aichi-cc.jp

Y. Ito

Department of Gastroenterological Surgery, Aichi Cancer Center Central Hospital, 1-1 Kanokoden, Chikusa-ku, Nagoya, Japan lapatinib, similar to the parental cells, in vitro. This growth inhibition of the Tmab-resistant cells by lapatinib was due to both G1 cell-cycle arrest and apoptosis induction via effective blockade of the PI3K/Akt and MAPK pathways. A preclinical study confirmed that the Tmab-resistant tumors are significantly susceptible to lapatinib.

Conclusion These results suggest that lapatinib has antitumor activity against the Tmab-resistant gastric cancer cell lines, and that these cell lines are useful for understanding the mechanism of Tmab resistance and for developing a new molecular therapy for Tmab-resistant HER2-positive gastric cancers.

Keywords HER2-positive gastric cancer - Cell lines · Trastuzumab resistance - Lapatinib · HER2 gene amplification

\section{Introduction}

In 2008, gastric cancer was the second and fifth most commonly diagnosed cancer in Japan and Europe, respectively [1]. Despite progress in therapeutic modalities, the survival rate of gastric cancer patients is still relatively poor when compared with those for other common cancers, such as colorectal and breast cancers. In gastric and gastroesophageal junction cancer patients, HER2 overexpression was observed in approximately 20 and $30 \%$ of the cases, respectively, most of which were of the histologically intestinal type. In gastric cancers, the major histological subtype is diffuse rather than intestinal, so HER2positive gastric cancers account for important members of intestinal type gastric cancer [2-5].

Trastuzumab is the first clinically approved therapeutic antibody against human epidermal growth factor receptor 2 
(HER2). It is now used worldwide as a standard therapy in HER2-positive metastatic breast cancer patients and as an adjuvant therapy in postoperative breast cancer patients [68]. Ouchi et al. [9] reported significant antitumor activity of trastuzumab in combination with chemotherapy in human HER2-positive gastric cancer cell xenograft models. More recently, an international randomized phase III trial (the TOGA study) demonstrated significantly prolonged overall survival with chemotherapy plus trastuzumab treatment compared with chemotherapy alone in HER2-positive gastric cancer patients, in a similar manner to breast cancer cases [10]. However, the response rate of trastuzumab was reportedly $47 \%$, and the median overall survival in patients with trastuzumab + chemotherapy was only 2.7 months longer than that in patients with chemotherapy alone, suggesting that primary trastuzumab resistance is prevalent and secondary (acquired) trastuzumab resistance is unavoidable. Thus, a potential strategy for overcoming such resistance is now urgently required. Reports are accumulating on mechanisms that can account for trastuzumab resistance in breast cancer cases, including autocrine production of EGF-like ligands, overexpression of the alternative IGF-IR pathway, and the production of P95HER2 and overexpression of MUC4 membrane-associated mucin glycoprotein [11-15]. To date, however, the molecular mechanism of trastuzumab-resistance in HER2positive gastric cancers is essentially unknown. This is largely because there are few HE2-positive gastric cancer cell lines [16, 17] and an absence of available trastuzumabresistant gastric cancer cell lines, in contrast to the situation for breast cancers.

HER2, a member of the HER family, can strongly interact with other HER receptors to form EGFR/HER2 and HER2/HER3 (HER4) heterodimers [18]. Therefore, HER2 is capable of constitutively transducing signals downstream to such as the Ras/mitogen-activated protein kinase (MAPK) and phosphatidylinositol-3-kinase (PI3K)/ protein kinase B pathways by upregulating HER2. Consequently, HER2 overexpression mediates multiple pathological responses, including a chemokine receptor, CXCR4-associated metastasis, and multidrug resistance due to activation of the PI3K/Akt pathway [19, 20]. Therefore, HER2-overexpressing gastric cancers are ideal targets for tyrosine kinase inhibitor (TKI) of HER2. Lapatinib is one such dual TKI that inhibits the phosphorylation of both EGFR and HER2, thereby interrupting the downstream MAPK and PI3K/Akt signaling pathways. Lapatinib was found to be clinically effective in patients with HER2-positive metastatic breast cancer that progressed after trastuzumab-based therapy [21, 22]. Recently, Kim et al. [23, 24] demonstrated that HER2 gene-amplified gastric cancer cell lines are also significantly sensitive to lapatinib. To date, however, no one has reported the lapatinib sensitivity of trastuzumab-resistant gastric cancer cell lines.

We previously established two HER2 gene-amplified gastric cancer cell lines (GLM-1 and GLM-4) from Japanese patients with liver metastasis and demonstrated the sensitivity of these cell lines to gefitinib [25, 26]. In the present study, we established trastuzumab-resistant gastric cancer cell lines from HER2 gene-amplified gastric cancer cell lines for the first time and demonstrated the lapatinib sensitivity of trastuzumab-resistant gastric cancer cells, implying the possibility that lapatinib may be a clinically useful agent against trastuzumab-resistant HER2-overexpressing gastric cancers.

\section{Materials and methods}

\section{Compounds}

Lapatinib (lapatinib ditossylate) for in vitro use was purchased from Santa Cruz Biotech (Santa Cruz, CA, USA). Trastuzumab (Herceptin, Genentech, South San Francisco, CA, USA), a humanized anti-HER2 monoclonal antibody, was purchased from Chugai Pharmaceuticals (Tokyo, Japan), and lapatinib (Tykerb) for preclinical study was obtained from GlaxoSmithKline (Philadelphia, PA, USA). Antibodies used were as follows. For western blotting analysis, mouse monoclonal antibodies to total human Erk1/2, phospho-Erk1/2 (Thr202/Tyr204), HER2 (Cell Signaling Technology, Danvers, MA, USA), MUC4 (Invitrogen, Carlsbad, CA, USA), and $\beta$-actin (Sigma-Aldrich, St. Louis, MO, USA) were used. Rabbit polyclonal antibodies to total Akt and phospho-Akt (Ser473) (Cell Signaling Technology) were used. Rabbit polyclonal antibody to HER2 (DAKO Cytomation, Glostrup, Denmark) was used for immunohistochemistry.

\section{Cell lines}

GLM-1 and GLM-4 cell lines were established in our laboratory from the liver metastasis of Japanese gastric cancer patients, as reported previously [25]. Trastuzumabresistant GLM-1 HerR2, GLM-1 HerR3, and GLM-4 HerR 2 cells were isolated after 2 or 3 cycles of in vivo selection, which consisted of twice-weekly intraperitoneal trastuzumab treatment for 4 weeks and subsequent culture of residual small peritoneal metastatic tumor (Fig. 1c). GLM-1 HerR2 and GLM-1 HerR3 cells show essentially the same characteristics. These cell lines were maintained in DMEM (Nissui Pharmaceutical Co., Ltd., Tokyo, Japan) supplemented with $10 \%$ fetal bovine serum (FBS) (GIB$\mathrm{CO}$, Grand Island, NY, USA), $100 \mathrm{U} / \mathrm{ml}$ penicillin, and 

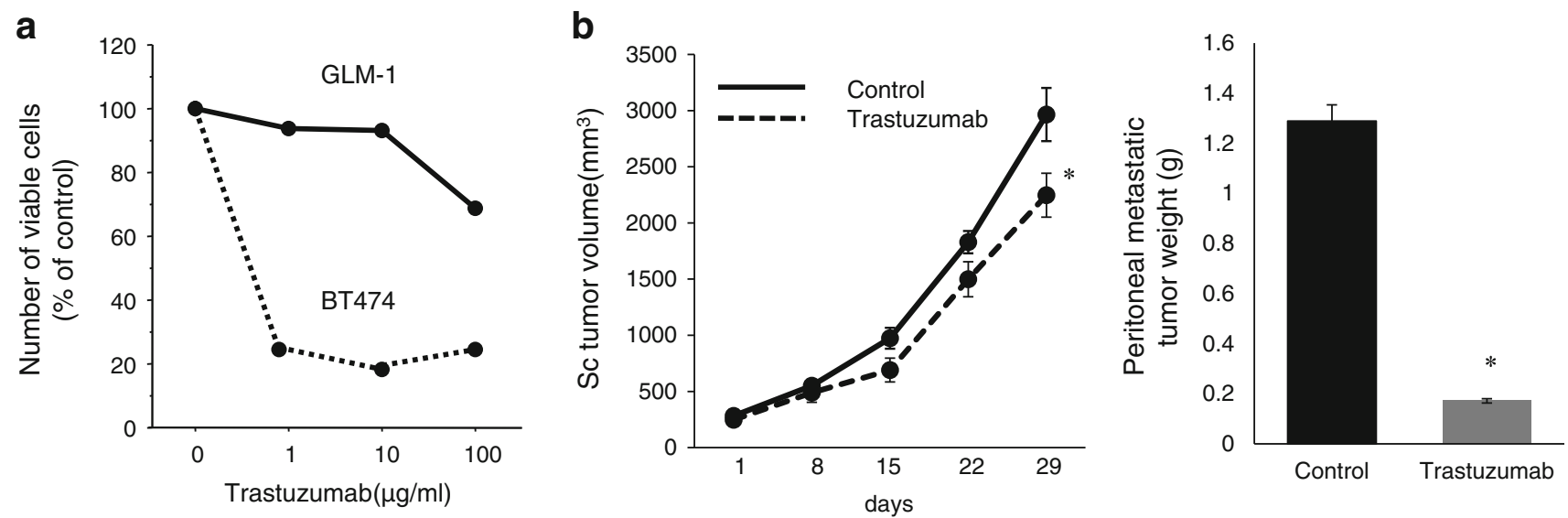

C
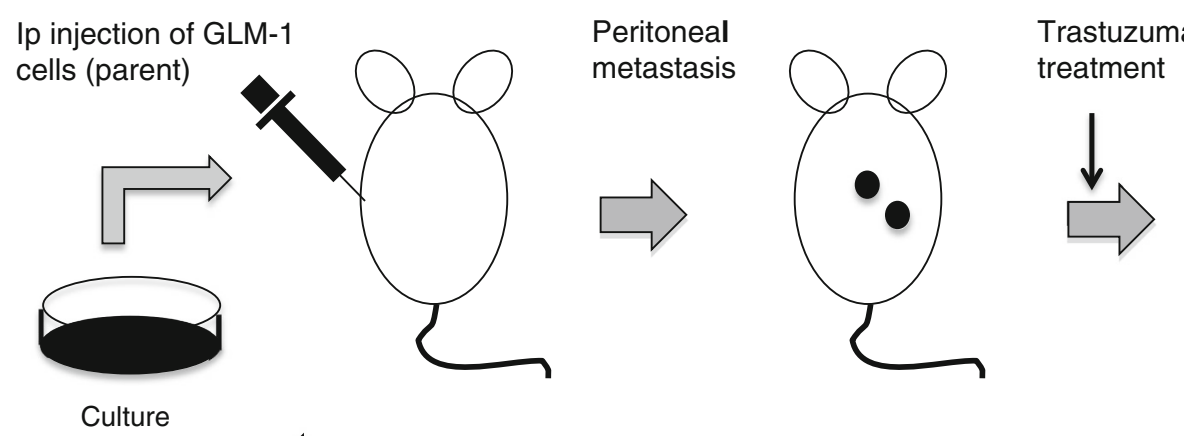

GLM-1 HerR1

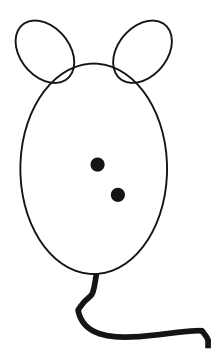

Remove small residual metastasis

Fig. 1 Isolation of trastuzumab-resistant HER2-positive gastric cancer cell lines by in vivo selection. a Comparison of growth inhibition of GLM-1 and BT474 cells by trastuzumab in vitro. b Effect of trastuzumab on the growth of subcutaneous (sc) tumor and intraperitoneal metastasis in nude mice. Trastuzumab was intraperitoneally (ip) injected into nude mice twice weekly for 4 weeks. $(P<0.05$ vs

$100 \mu \mathrm{g} / \mathrm{ml}$ streptomycin in plastic dishes (Falcon, Franklin Lakes, NJ, USA), and incubated at $37{ }^{\circ} \mathrm{C}$ in $5 \% \mathrm{CO}_{2}$.

In vitro cell growth assay

Cells were harvested with trypsin/EDTA, plated at $1 \times 10^{4}$ cells/96-well plastic plate in DMEM supplemented with $10 \% \mathrm{FBS}$, and then treated with increasing doses of trastuzumab $(0,1,10$, and $100 \mu \mathrm{g} / \mathrm{ml})$ or lapatinib $(0.1,1.0$, and $10 \mu \mathrm{M}$ ) on day 1 . The number of viable tumor cells was counted on day 3 with a hemocytometer in triplicate.

\section{Cell cycle analysis by flow cytometer}

To determine the percentage of cells at various phases of the cell cycle, exponentially growing cells were treated for $24 \mathrm{~h}$ with trastuzumab or lapatinib at a concentration of $100 \mu \mathrm{g} / \mathrm{ml}$ or $10 \mu \mathrm{M}$, respectively, and cells were analyzed control). c Schematic representation of the procedure for isolating trastuzumab-resistant cells from parental cells. Trastuzumab-resistant cells were isolated after 2 or 3 cycles of in vivo selection, which consisted of trastuzumab treatment for 4 weeks and subsequent culture of residual small metastatic tumor in the peritoneal cavity in vitro

for nuclear DNA after propidium iodide staining using Cycletest Plus kit (Becton Dickinson, San Jose, CA, USA) according to the manufacturer's instructions. Flow cytometric analysis was done in FACSCalibur (Becton Dickinson Biosciences, San Diego, CA, USA). Data collected from 10,000 cells for each experiment were analyzed by the ModFit software package (Verity Software House, Topsham, ME, USA).

\section{Apoptosis assay}

Apoptosis induced by trastuzumab and lapatinib was quantitated using an Annexin V-FITC apoptosis detection kit (Biovision, Mountain View, CA, USA). Cells were plated at $1 \times 10^{5}$ cells $/ 6 \mathrm{~cm}$ plastic plate in DMEM $+10 \%$ FBS and treated with trastuzumab $(100 \mu \mathrm{g} / \mathrm{ml})$ or lapatinib $(10 \mu \mathrm{M})$ for $42 \mathrm{~h}$. Cell-surface annexin $\mathrm{V}$ binding and propidium iodide (PI) uptake were detected by a flow cytometer. 
Western blot analysis

The monolayer culture cells were maintained on $60 \mathrm{~mm}$ dishes in medium supplemented with $10 \%$ FBS. Cells were exposed to trastuzumab $(10,100 \mu \mathrm{g} / \mathrm{ml})$ and lapatinib $(1.0$ and $10 \mu \mathrm{M})$ for $24 \mathrm{~h}$ at $37^{\circ} \mathrm{C}$. Cells were then lysed at $4{ }^{\circ} \mathrm{C}$ in lysis buffer $(10 \mathrm{mM}$ Tris- $\mathrm{HCl}, \mathrm{pH} 7.5,150 \mathrm{mM}$ $\mathrm{NaCl}, 1 \%$ Triton X-100, $1 \mathrm{mM}$ EDTA, and complete Protease Inhibitor Cocktail). The protein concentration was determined by Lowry assay (DC Protein Assay; Bio-Rad, Hercules, CA, USA), and 50- $\mu \mathrm{g}$ cell aliquots were directly lysed in Laemmli sample buffer and separated by SDSPAGE under reducing conditions, before being transferred to a PVDF membrane (Bio-Rad) and immunoblotted with antibodies. Bound antibodies were visualized using SuperSignal West Pico (or Dura) chemiluminescence substrate (Thermo Scientific, Waltham, MA, USA).

\section{FISH analysis}

Amplification of the c-erbB-2 gene was determined by a dual-color FISH method using a Passvision HER-2 DNA probe kit (Vysis Inc., Downers Grove, IL, USA) according to the manufacturer's protocol. The HER-2/neu-Spectrum Orange probe contains a DNA sequence specific for the c-erbB-2 human gene locus and hybridizes to region 17q11.2-q12 of the human chromosome. The CEP 17 green probe that hybridizes to the D17Z1 locus (centromere region of chromosome 17) was used as a control. The nucleus was counterstained with 4',6-diamidino-2-phenylindole (DAPI). The slides were observed under a BX60 fluorescence microscope equipped with a digital camera (DP50, Olympus, Tokyo, Japan). A cell was considered to show amplification when a definite cluster of more than 4 signals for HER2 was present.

\section{Immunohistochemical analysis}

Subcutaneous tumors in nude mice were removed and fixed in $10 \%$ buffered formalin for $24 \mathrm{~h}$. Formalin-fixed and paraffin-embedded sections $(4 \mu \mathrm{m})$ were used for immunohistochemistry. For antigen retrieval, the sections were treated with microwaves at $98{ }^{\circ} \mathrm{C}$ for $10 \mathrm{~min}$. After blocking nonspecific reactions, the sections were incubated at $4{ }^{\circ} \mathrm{C}$ overnight with antibodies to HER2 and MUC4 with optimal dilution. After washing with PBS, the sections were incubated with biotinylated second antibodies for $30 \mathrm{~min}$. The sections were washed again with PBS, then incubated with streptavidin-peroxidase complex (Vectastain ABC kit, Vector Laboratories, Burlingame, CA, USA) for $60 \mathrm{~min}$. The chromogen was developed with $0.01 \%$ diaminobenzidine (DAB), and the sections were counterstained with Meyer's hematoxylin. Immunohistochemistry for
HER2 as described above is similar to HercepTest (Dako Cytomation) in that they use the same polyclonal antibody and estimation system. Tumors with membrane staining scores of $2+$ and $3+$ according to the HercepTest criteria were evaluated as positive.

\section{Tumor xenograft studies}

Growing cultured cells were harvested with trypsin-EDTA and washed with PBS, and then $5 \times 10^{6}$ cells in $0.2 \mathrm{ml}$ PBS were injected subcutaneously into the left abdominal flanks of 6- to 8-week-old male nude mice of the KSN strain (Shizuoka Laboratory Animal Center, Hamamatsu, Japan). Mice $(n=5)$ were intraperitoneally injected with trastuzumab $(20 \mathrm{mg} / \mathrm{kg} / \mathrm{day}$, twice weekly for 4 weeks $)$ and orally administered lapatinib $(150 \mathrm{mg} / \mathrm{kg} /$ day 5 times per week for 4 weeks). In the control groups, mice were administered the vehicle. The maximum tumor diameter $(L)$ and the diameter perpendicular to that axis $(W)$ were measured every 5 days. Tumor volume was estimated by the following formula: $L \times W \times W \times 1 / 2$. All experiments were carried out with the approval of the Institutional Ethical Committee for Animal Experiments of the Aichi Cancer Center Research Institute, and met the standard as defined by the UKCCR guidelines [27].

\section{Statistical analysis}

The statistical significance of any difference in corresponding data between treatment groups was determined by applying Student's $t$ test. A $P$ value of $<0.05$ was considered significant.

\section{Results}

We first compared growth inhibition by trastuzumab between the HER2 gene-amplified gastric and breast cancer cell lines. An in vitro study showed that GLM-1 cells were less sensitive to trastuzumab than the breast cancer cell line (BT474) was (Fig. 1a). In the GLM-1 sc tumor model in nude mice, trastuzumab significantly suppressed sc tumor growth, but the residual tumor after therapy was still large in size (Fig. 1b left). In contrast, the growth of peritoneal metastasis was markedly inhibited by trastuzumab, and the remaining tumor was very small in size (2-3 mm in diameter) (Fig. 1b right), indicating higher trastuzumab sensitivity of the peritoneal metastasis than the sc tumor. Based on these findings, to isolate the trastuzumab-resistant variant cell line, we adopted an in vivo selection method using a peritoneal metastasis model. We first injected three HER2 gene-amplified gastric cancer cell lines (GLM-1, GLM-4, and NCI-N87) intraperitoneally 
a
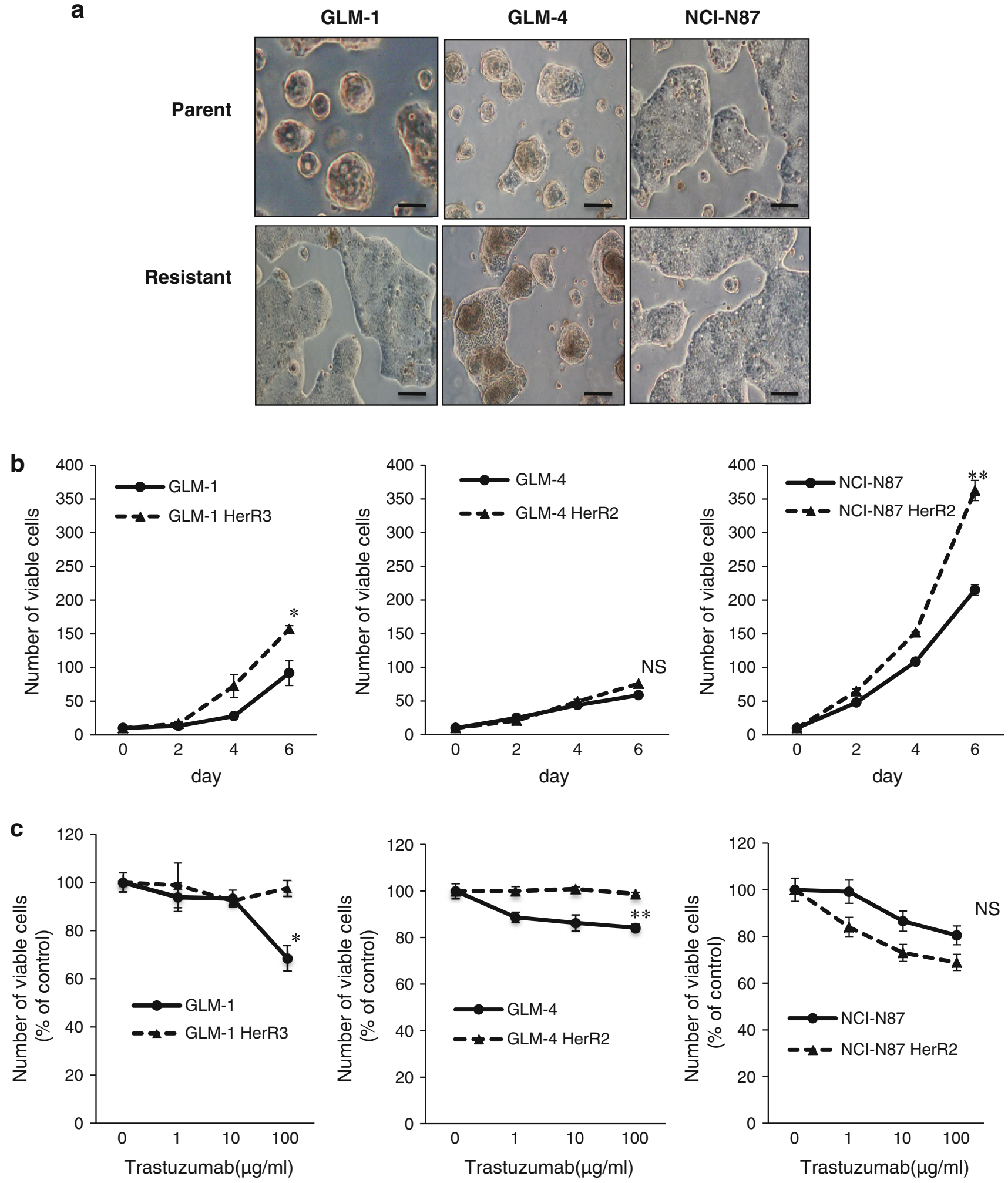

Fig. 2 Characteristics of trastuzumab-resistant GLM-1, GLM-4, and NCI-N87 cells. a Comparison of the cultured cell morphologies of parental cells and trastuzumab-resistant cells (GLM-1HerR3, GLM4HerR2, and NCI-N87HerR2). Bars $100 \mu \mathrm{m}$. b Comparison of the in vitro growth of parental cells and trastuzumab-resistant cells. c Effect of trastuzumab on the growth of parental cells and trastuzumab-resistant cells. $* P<0.05, * * P<0.01$ 
and started weekly intraperitoneal trastuzumab treatment on day 4 for 4 weeks. After treatment, we sacrificed the mice and removed residual small peritoneal metastases and cultured them. By repeating this procedure for 2 or 3 cycles, we isolated trastuzumab-resistant variant cell lines (GLM-1 HerR2, GLM-1 HerR3, GLM-4 HerR2, and NCIN87 HerR2) (Fig. 1c).

Trastuzumab-resistant cell lines showed a more flattened morphology and faster growth than parental cells with a multilayered growth pattern in vitro (Fig. 2a, b). Furthermore, parental cells expressed more sucrose-isomaltase than trastuzumab-resistant cells (data not shown), suggesting that differences in the growth and morphology may be related to a difference in the intestinal differentiation of HER2-gene-amplified gastric cancer cells. However, the histology of sc tumors in nude mice was tubular or papillary adenocarcinoma, and was essentially the same for GLM-1 and GLM-4 parental and trastuzumab-resistant
Fig. 4 HER2 protein expression and gene amplification in GLM-1, GLM-4, and NCI-N87 parental and trastuzumab-resistant cells. a HER2 expression as determined by immunohistochemistry of subcutaneous tumor xenografted in nude mice. Note the strong membranous staining in all of the cells. b HER2 gene amplification determined by dual-color FISH of cultured cells. Cluster pattern (red) gene amplification was observed in all cell types. c Flow cytometric analysis of HER2 expression of cultured cells. d Flow cytometric analysis of the binding of FITC-labeled trastuzumab to cultured cells. In this experiment, we used GLM-1 HerR3 cells as trastuzumabresistant GLM-1 cells

tumors at the HE level (data not shown). GLM-1 and GLM-4 cells were modestly but significantly sensitive to trastuzumab, whereas trastuzumab-resistant cells were refractory to trastuzumab in vitro, except for NCI-N87 cells (Fig. 2c). Subcutaneous tumor growth in nude mice of parental GLM-1, GLM-4 and NCI-N87 cells was significantly suppressed by the trastuzumab treatment, whereas GLM-1HerR3 and GLM-4HerR2 tumor growth was not a

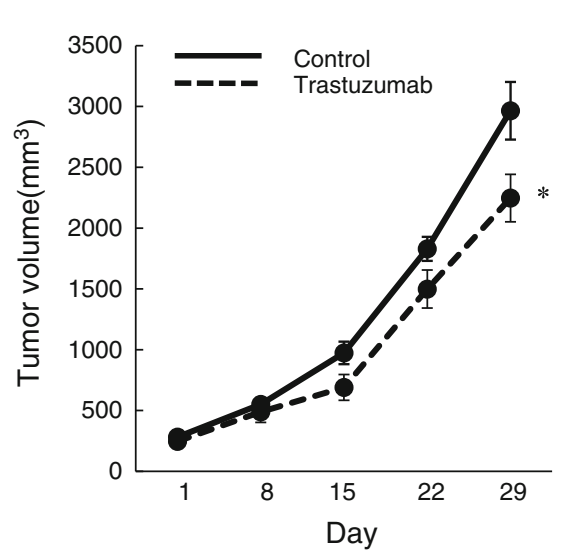

b

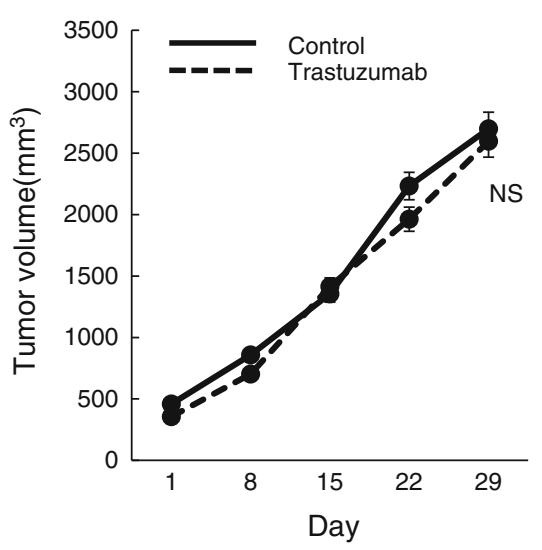

GLM-4

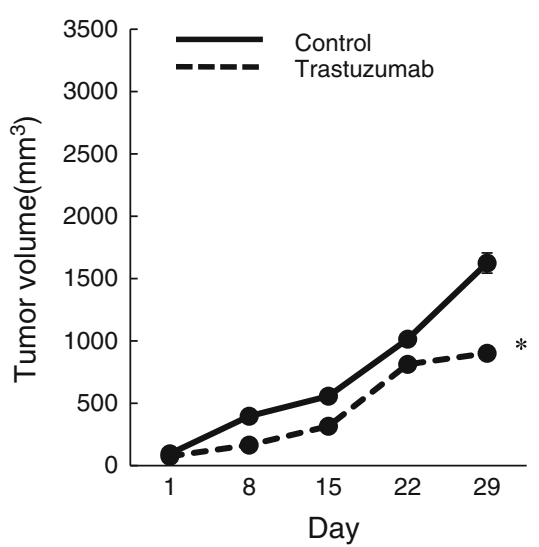

GLM-4 HerR2

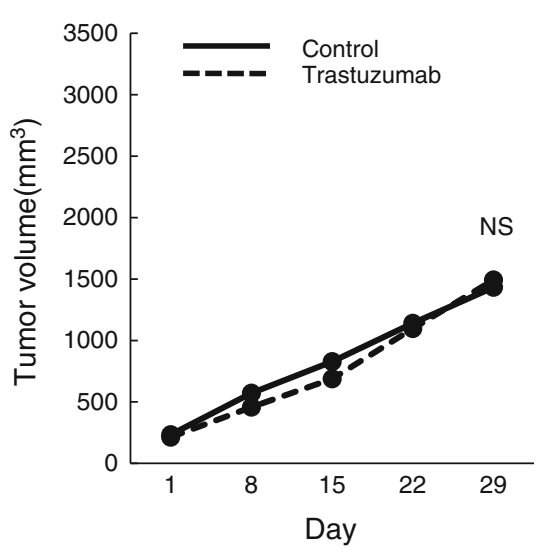

NCI-N87

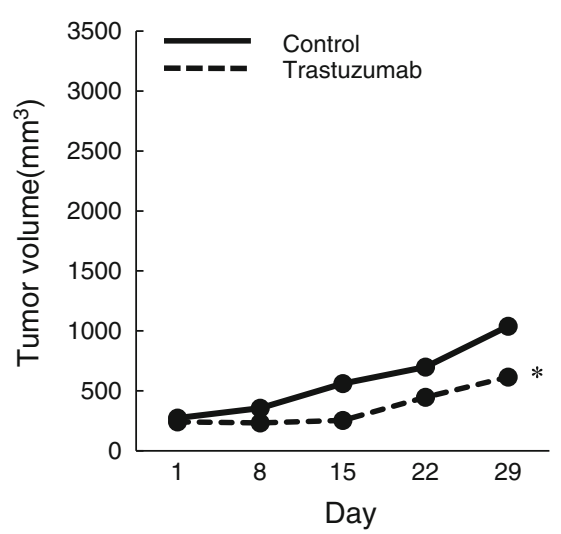

NCI-N87 HerR2

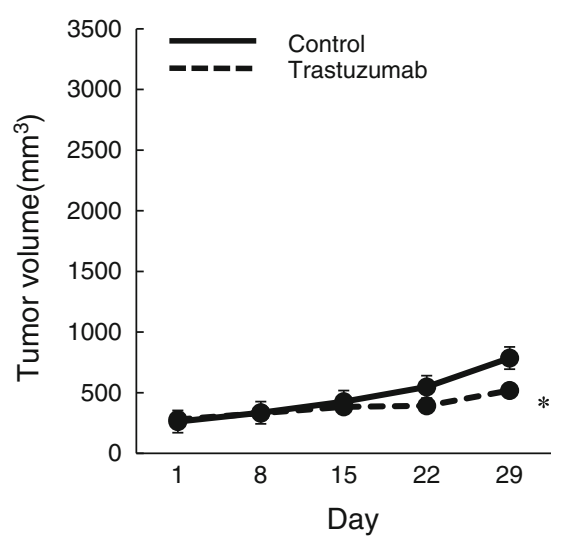

Fig. 3 Trastuzumab sensitivities of GLM-1, GLM-4, and NCI-N87 parental and trastuzumab-resistant cells in subcutaneous tumor xenografted into nude mice. a Parental tumor cells and b trastuzumab-resistant cells were injected subcutaneously into nude mice, and intraperitoneal treatment with trastuzumab was done at a dose of $20 \mathrm{mg} / \mathrm{kg} /$ day, twice weekly, for 4 weeks. $* P<0.05$ (vs control). NS not significant. Bars SE 


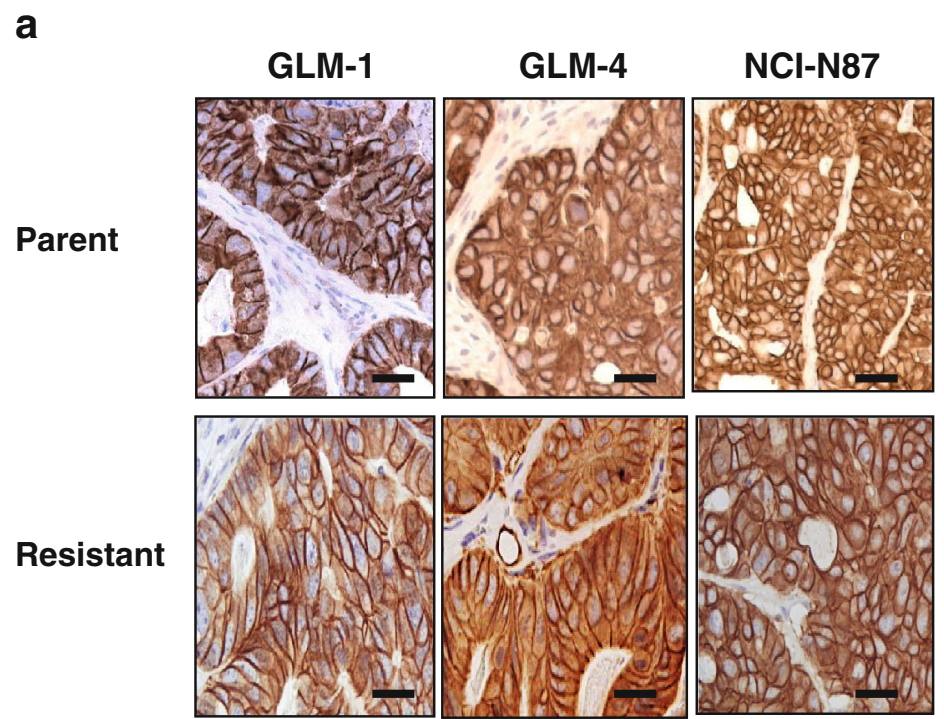

b

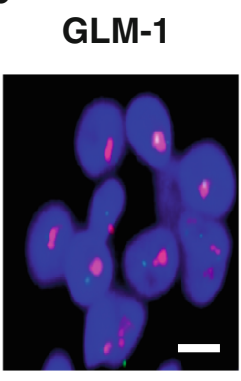

GLM-4 NCl-N87
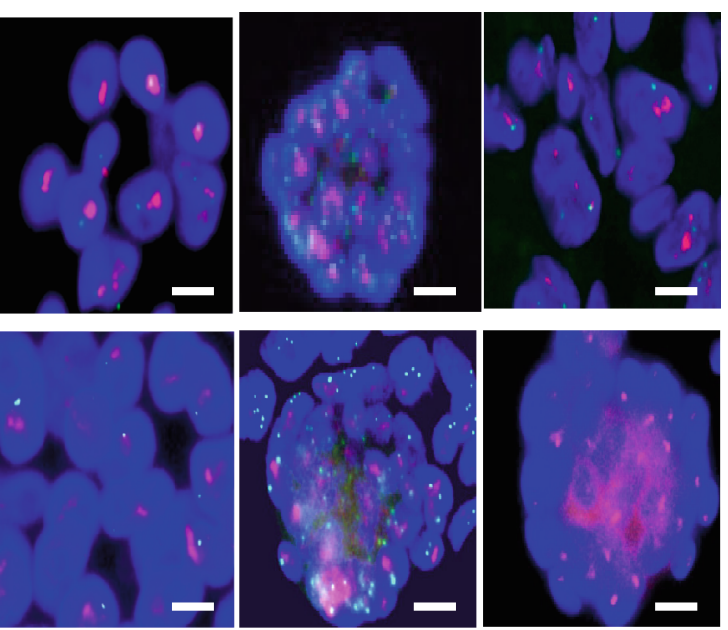

C
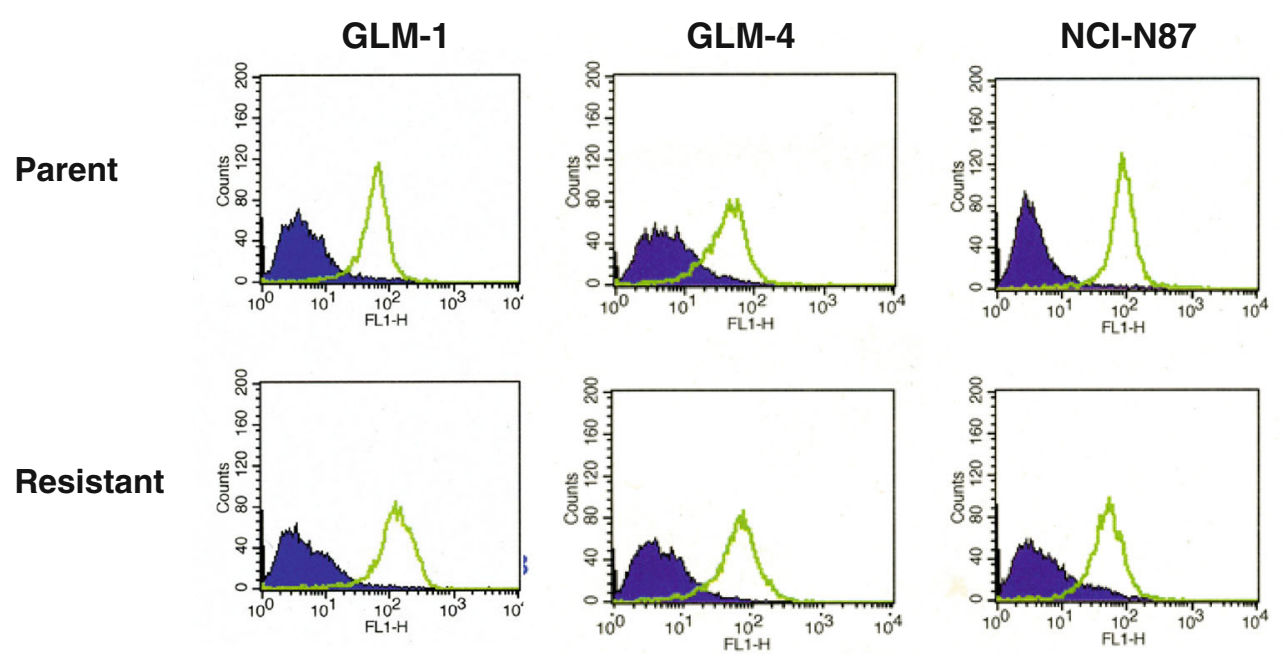

d
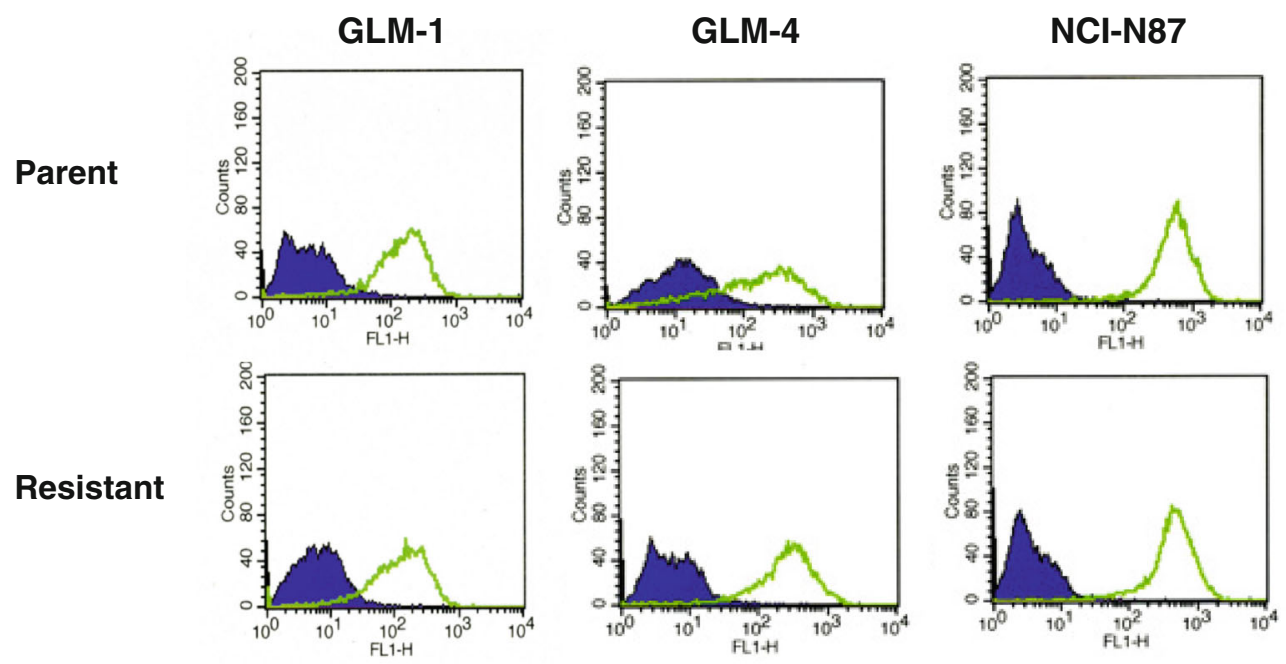
(Fig. 3a, b), confirming the trastuzumab resistance of GLM-1HerR2, 3 and GLM-4HerR2 cells both in vitro and in vivo. In contrast, NCI-N87 HerR2 cells were still found to be sensitive to trastuzumab both in vitro and in vivo.

Immunohistochemical analysis of the HER2 expression of xenografted tumor demonstrated similar strong HER2 protein expression on the cell surface in both the parental and resistant cells (Fig. 4a). FISH analysis also showed that both parental and resistant cells had similar levels of cluster-type HER2 gene amplification (Fig. 4b). Flow cytometric analysis revealed that HER2 expression of these trastuzumab-resistant cells was not significantly different from that of parental cells (Fig. 4c). Furthermore, the binding of trastuzumab to the tumor cell surface, as determined by the binding of Alexa488-labeled trastuzumab, was similar for the parental and resistant cells (Fig. 4d), suggesting that trastuzumab resistance was not due to the downregulation of HER2 expression and decreased HER2 binding to the cells.

a

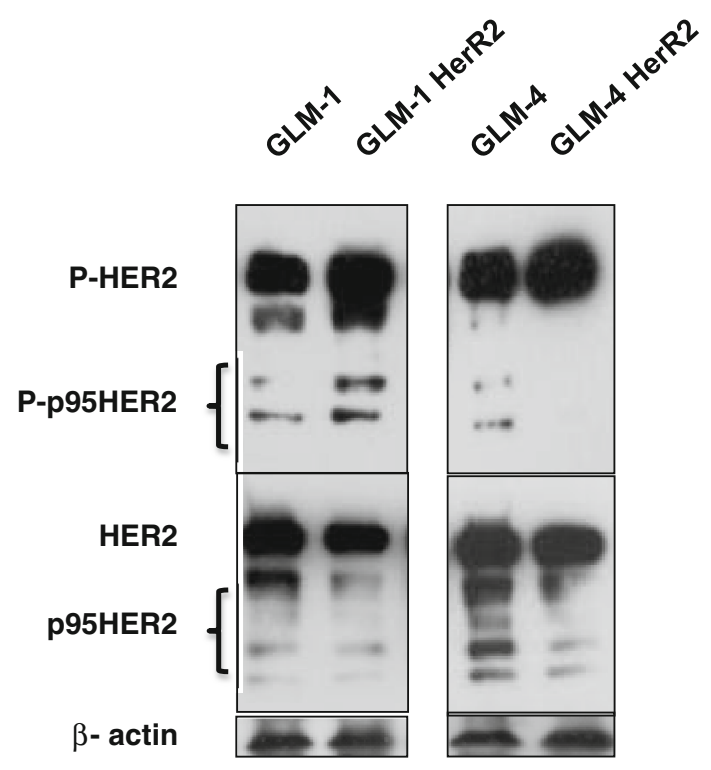

b

MUC4

$\beta$ - actin
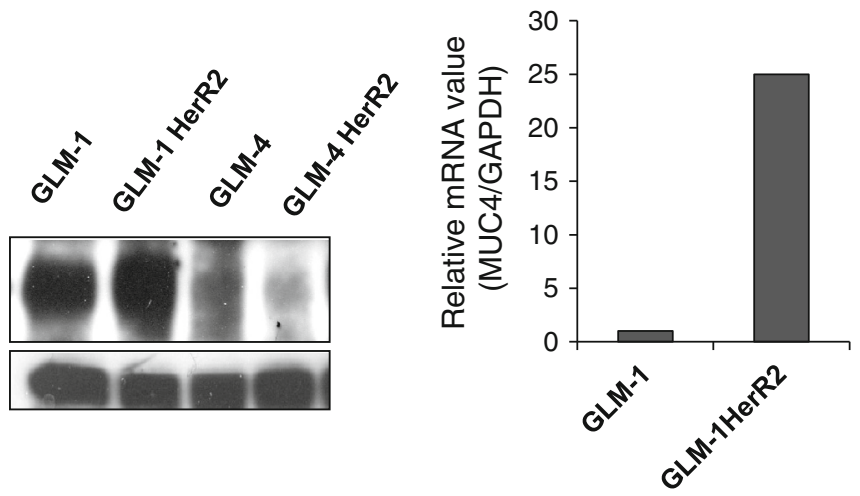

C

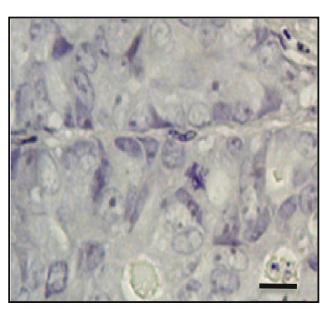

GLM-4

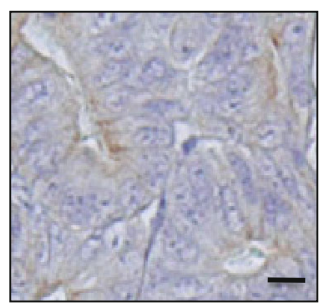

GLM-4 HerR2

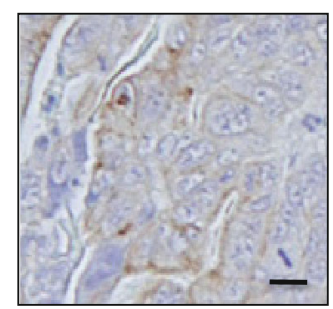

GLM-1 HerR2

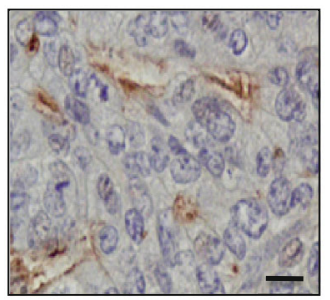

immunohistochemical analysis of MUC4 protein expression. c qRTPCR analysis of MUC4 mRNA expression of cultured cells. Bars $10 \mu \mathrm{m}$
Fig. 5 p95HER2 and MUC4 expression in GLM-1 and GLM-4 parental and trastuzumab-resistant cells. a Western blot analysis of p95HER2 expression in cultured cells. b Western blot and
Fig. 6 Lapatinib sensitivities of GLM-1 and GLM-4 parental and trastuzumab-resistant cells. After treatment with trastuzumab and lapatinib for $24 \mathrm{~h}$, cells were lysed and subjected to western blot

We then examined the expression of p95HER2 and MUC4, which are reportedly involved in the trastuzumab resistance of HER2-positive breast cancer cell lines, at the protein and mRNA level. p95HER2 consists of several components with MWs ranging from 95 to $115 \mathrm{kDa}$, and there was no substantial increase in p95HER2 protein expression or their phosphorylation in GLM-1- and GLM4-resistant cells compared with parental cells (Fig. 5a). MUC4 expression increased on the surfaces of GLM-1 trastuzumab-resistant cells at the protein and mRNA levels, whereas it decreased in the GLM-4 and GLM-4HerR2 cells 


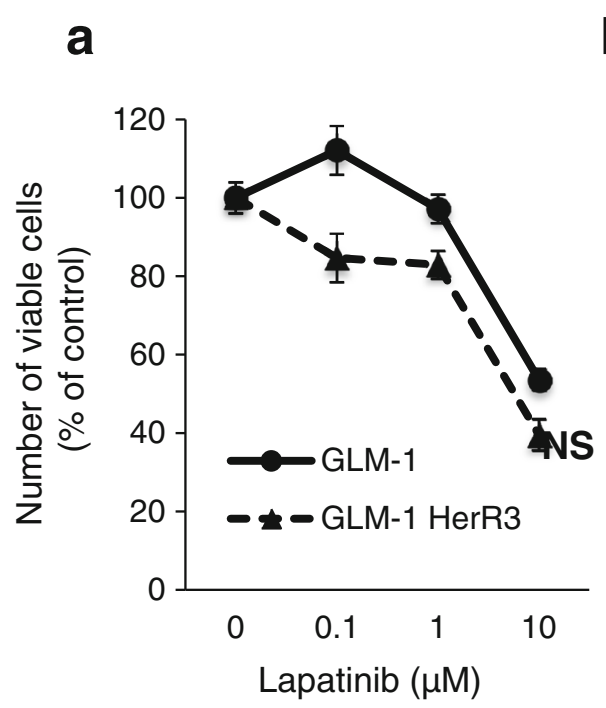

b

GLM-1

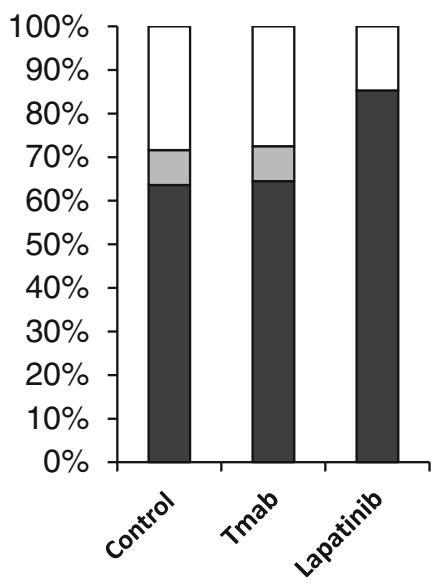

GLM-1 Her R3

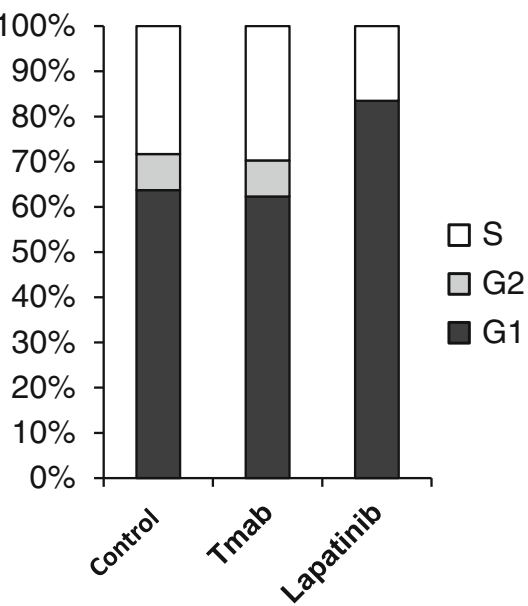

C

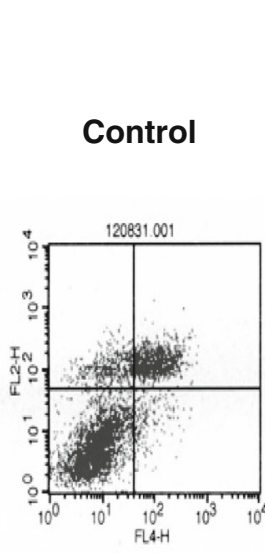

\section{GLM-1 HerR3 cell}
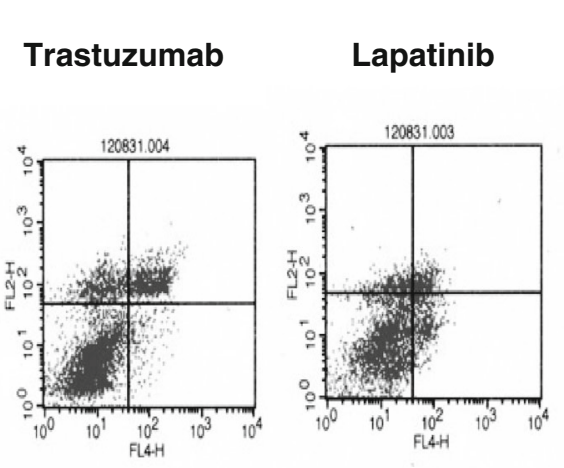

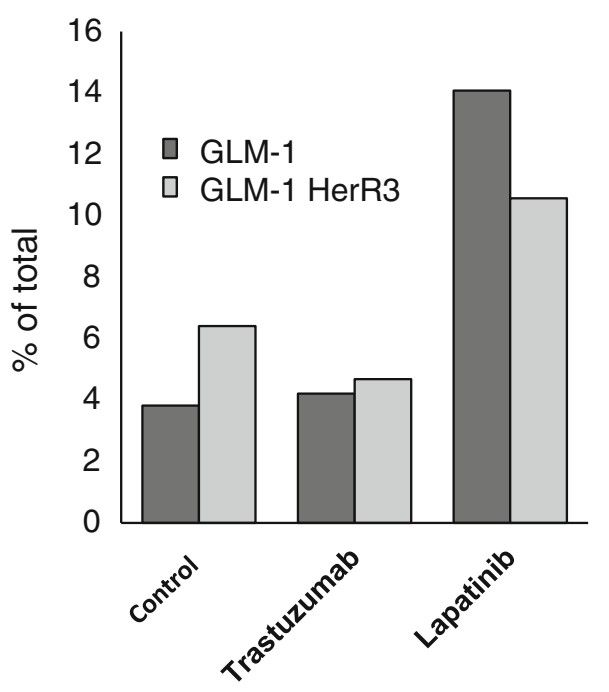

d

GLM-1

GLM-1 HerR3

Trastuzumab $(\mu \mathrm{g} / \mathrm{ml})$
Lapatinib $(\mu \mathrm{M})$
P-HER2
HER2
P-Akt
Akt
P-Erk
Erk
$\beta-A c t i n$

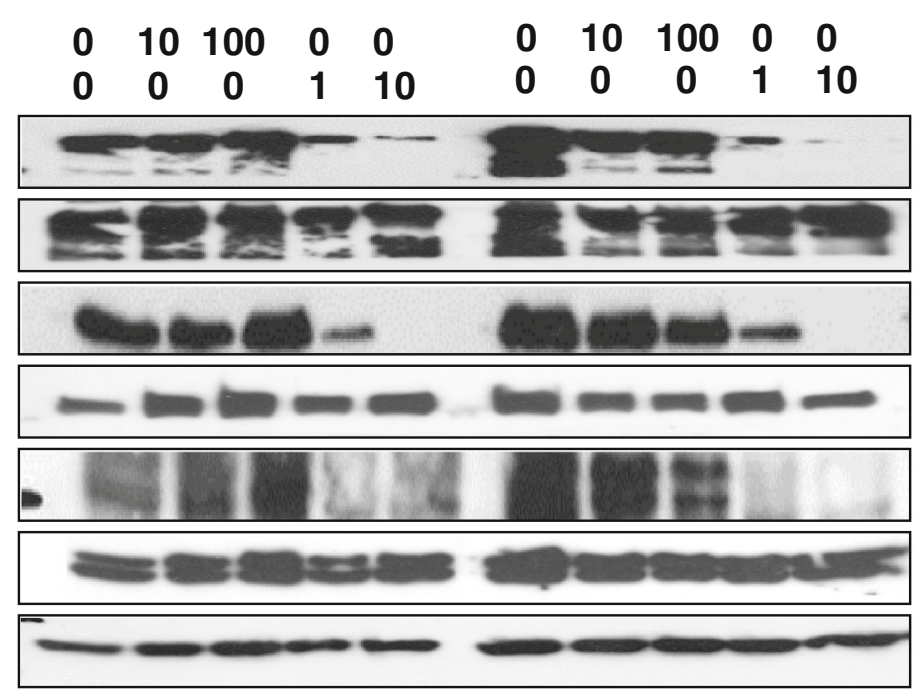


a

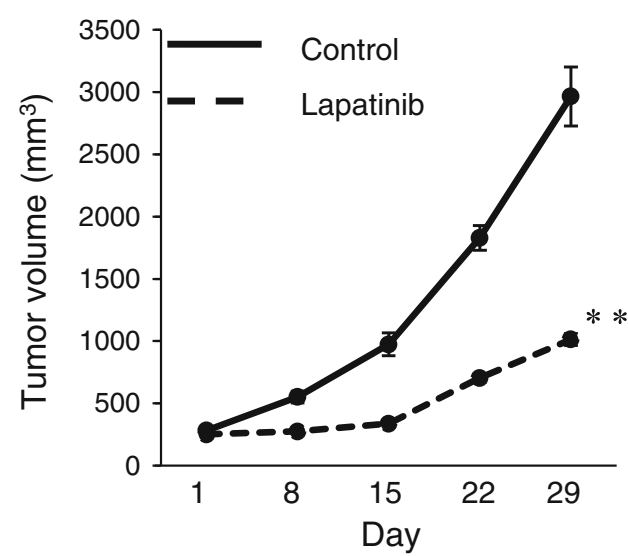

GLM-1

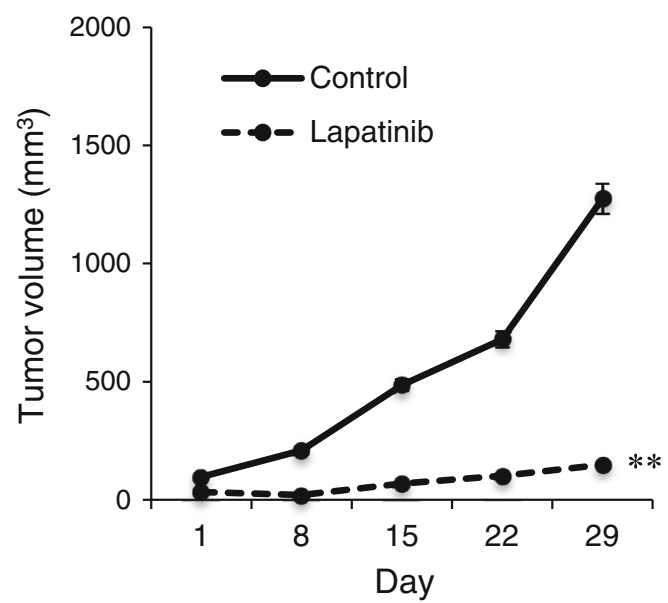

GLM-4

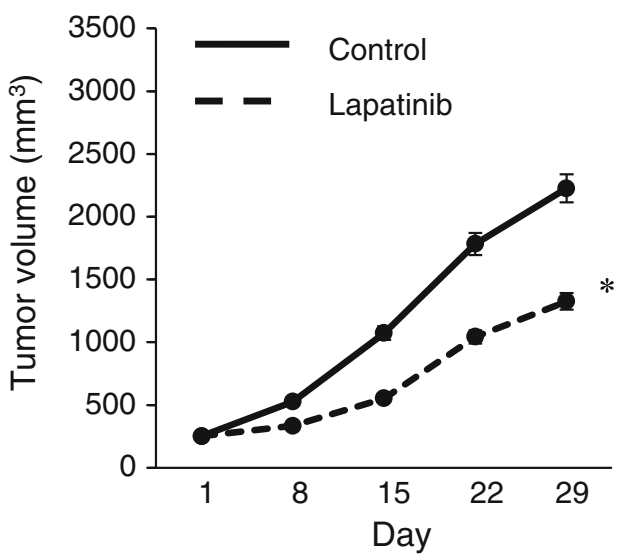

GLM-1 HerR2

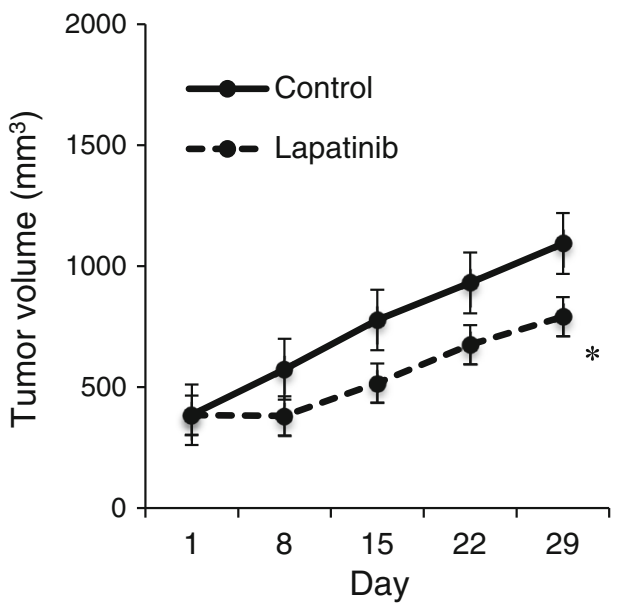

GLM-4 HerR2

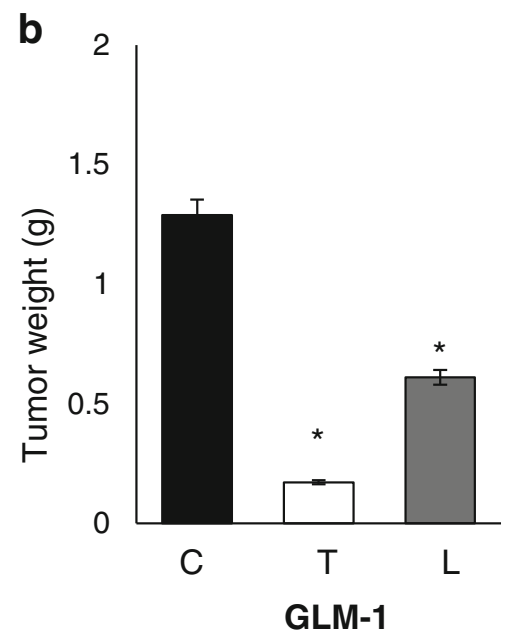

Fig. 7 Antitumor and antimetastatic effects of trastuzumab and lapatinib on xenografted tumors in nude mice. a Effects of lapatinib on the growth of GLM-1 and GLM-4 parental and trastuzumabresistant subcutaneous tumors in nude mice. b Effects of trastuzumab and lapatinib on the growth of GLM-1 parental and trastuzumabresistant peritoneal metastasis. Tumor cells were injected subcutaneously or intraperitoneally into nude mice $(n=4-6)$, and lapatinib

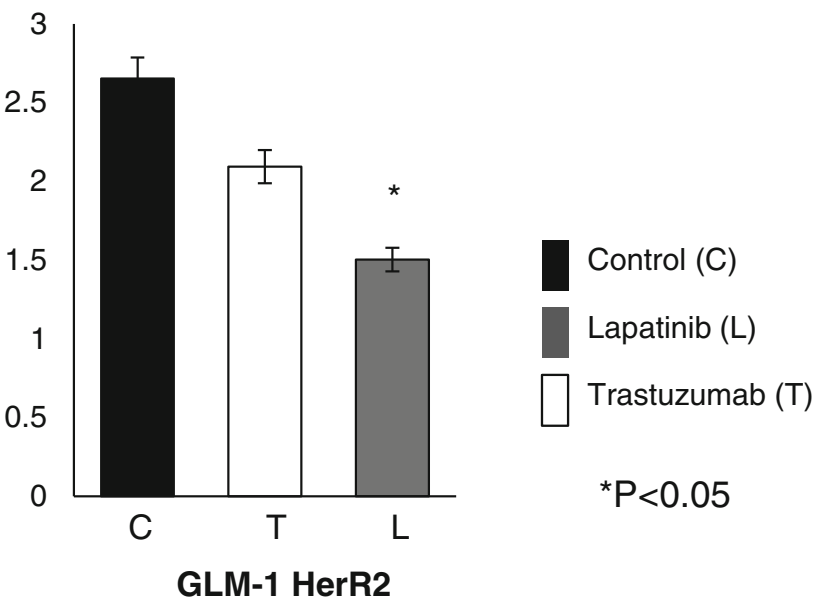

( $150 \mathrm{mg} / \mathrm{kg} / \mathrm{day}$, oral administration, five times per week for 4 weeks) or trastuzumab $(20 \mathrm{mg} / \mathrm{kg} / \mathrm{day}$, intraperitoneal injection, twice a week for 4 weeks) was administered. Photographs of representative peritoneal metastasis (arrows) in nude mice treated by trastuzumab or lapatinib. ${ }^{*} P<0.05,{ }^{*} P<0.01$ (vs control). $N S$ not significant. Bars SE 
(Fig. 5b, c), indicating different expression in the different cell lines.

NCI-N87 HerR2 cells were still found to be sensitive to trastuzumab after in vivo selection. So, thereafter, we only examined GLM-1 and GLM-4 trastuzumab-resistant cells to further analyze their susceptibility to lapatinib. In vitro and in vivo studies of the lapatinib sensitivities of these trastuzumab-resistant cell lines demonstrated that, despite trastuzumab-resistance, GLM-1HerR3 cells remained lapatinib-sensitive to a degree comparable to parent cells (Fig. 6a). Cell cycle analysis showed that the percentages (\%) of the S/G2/G1 phases of control GLM-1 cells, tastuzumab-treated GLM-1 cells, and lapatinib-treated GLM-1 cells were $28.5 / 8.0 / 63.5 \%, 27.5 / 8.0 / 64.5 \%$, and $14.7 / 0 /$ $85.3 \%$, respectively. On the other hand, the percentages (\%) of the S/G2/G1 phases of the control GLM-1HerR3, trastuzumab-treated GLM-1HerR3, and lapatinib-treated GLM-1HerR3 cells were 28.2/8.0/63.8\%, 29.7/8.0/ $62.3 \%$, and $16.5 / 0 / 83.5 \%$, respectively, indicating that G1 cell-cycle arrest was induced in both cells to similar extents (Fig. 6b). Annexin V assay revealed that apoptosis induction, as evaluated by determining the annexin $+/ \mathrm{PI}-$ fractions of control GLM-1, trastuzumab-treated GLM-1, and lapatinib-treated GLM-1 cells were 3.93, 4.12, and $14.2 \%$, respectively, whereas those of the GLM-1HerR3 cells were $6.36,4.34$, and $10.49 \%$, respectively (Fig. 6c), implying that apoptosis was induced by lapatinib to similar extents for the two cells.

Effects of trastuzumab and lapatinib on the downstream signaling pathways of GLM-1 cells and GLM-1HerR3 cells were examined by western blotting. Trastuzumab had no significant inhibitory effects on the phosphorylation of HER2, Akt, and Erk, whereas lapatinib inhibited the PI3K/ Akt and MAPK signaling pathways in both parental and trastuzumab-resistant cell lines to similar extents (Fig. 6d), consistent with the above results obtained from cell cycle analysis and apoptosis induction. Similar levels of growth inhibition, induction of G1 cell-cycle arrest and apoptosis, and inhibition of signal transduction by lapatinib were observed in GLM-4 cells and GLM-4HerR2 cells (data not shown).

Furthermore, we compared antitumor and antimetastatic effects of trastuzumab and lapatinib in GLM-1 (GLM-4) parental and trastuzumab-resistant cells. While there was no apparent inhibition of sc tumor growth by trastuzumab (Fig. 3b), lapatinib significantly inhibited sc tumor growth in trastuzumab-resistant GLM-1 and GLM-4 cells (Fig. 7a). In a peritoneal metastasis model, which was only applicable for the GLM1-HeR2 (3) cells because of the low metastatic potential of GLM-4 cells, trastuzumab and lapatinib strongly inhibited peritoneal metastasis in parental GLM-1 cells, whereas only lapatinib significantly inhibited peritoneal metastasis of GLM-1HerR3 cells (Fig. 7b), indicating that lapatinib has antimetastatic potential for trastuzumab-resistant cells.

\section{Discussion}

In the present study, we successfully isolated two novel trastuzumab-resistant variant cell lines from HER2 geneamplified parental gastric cancer cell lines. These cell lines are unique for the following reasons. (1) Although trastuzumab-resistant HER2-positive breast cancer cell lines such as BT474 and SKBR-3 cells are now available [28, 29], no HER2-positive, trastuzumab-resistant gastric cancer cell lines have been reported. Our cell lines are thus the first trastuzumab-resistant gastric cancer cell lines established by the new in vivo selection method. (2) HER2 gene-amplified gastric cancer cell lines are only modestly susceptible to trastuzumab in vitro, unlike the HER2positive breast cancer cell line, in which G1 cell-cycle arrest is induced by trastuzumab via decreased phosphorylation of Akt [11]. Therefore, the mechanisms of trastuzumab resistance in HER2-positive gastric and breast cancer cell lines may differ; for instance, antibody-dependent cellular cytotoxicity (ADCC) may be more important than the blockade of signal transduction in the former. (3) GLM-1 and GLM-4 cell lines derived from Japanese gastric cancer patients acquired trastuzumab resistance more markedly than NCI N-87 cells, which are derived from Caucasian HER2-positive gastric cancer patients after repetitive trastuzumab treatment. Consistent with these preclinical findings, a subset analysis of the TOGA study in which only Japanese patients were enrolled showed that the survival of patients treated with trastuzumab therapy was improved but the efficacy of trastuzumab was less than that seen in the worldwide full-set analysis [30]. These findings suggest the possibility of racial differences in the trastuzumab sensitivities of HER2-positive gastric cancers derived from Japanese and Western patients. Therefore, these HER2 gene-amplified, trastuzumab-resistant gastric cancer cell lines would be very useful preclinical models for understanding the detailed mechanism of acquired trastuzumab resistance, as well as for developing a new molecular targeting therapy to overcome the trastuzumab resistance of HER2 gene-amplified gastric cancer patients.

Interestingly, these trastuzumab-resistant gastric cancer cell lines were still sensitive to lapatinib to a similar extent to the parental cells. Lapatinib is a dual TKI that inhibits the phosphorylation of both HER2 and EGFR, thereby interrupting the downstream signaling pathways such as the MAPK and PI3K/Akt pathways. Lapatinib was reportedly active in women with HER2-positive metastatic breast cancer that progressed after trastuzumab therapy [21, 22]. Therefore, HER2 gene-amplified gastric cancer is also 
likely to respond to lapatinib. Although previous studies demonstrated that HER2 gene-amplified gastric cancer cell lines show significant sensitivity to lapatinib [23, 24], the antitumor activity of lapatinib against trastuzumab-resistant gastric cancer cell lines has remained unknown. In the present study, we first demonstrated that lapatinib significantly inhibits the growth of trastuzumab-resistant GLM-1 cells, just as it does for parental GLM-1 cells. This growth inhibition of GLM-1HerR3 cells by lapatinib was achieved through G1 cell-cycle arrest and apoptosis by inhibiting the phosphorylation of HER2 and downstream Akt and Erk, just as for the parental GLM-1 cells. Furthermore, significant antitumor and antimetastatic effects of lapatinib on GLM-1HerR3 cells were observed in the nude mouse xenograft model. Similar levels of growth inhibition by lapatinib was also observed in vitro and in vivo in GLM-4 HerR2 cells and parental GLM-4 lines. These results strongly suggest that lapatinib alone or in combination with trastuzumab (or chemotherapy) would be a potential new targeted therapy for metastatic HER2 gene-amplified gastric cancer patients with acquired trastuzumab resistance. Several clinical trials of lapatinib in combination with chemotherapy against HER2-positive gastric cancers are now ongoing. Based on our present findings, improved overall and progression-free survival rates would be expected in HER2-positive, trastuzumab-resistant gastric cancer patients treated with lapatinib, in addition to trastuzumab-sensitive patients.

There are several possible mechanisms for the acquired trastuzumab resistance of these gastric cancer cell lines, based on reports of studies of HER2-positive breast cancer cell lines. In the present study, we partially examined following three possibilities. First, the downregulation of HER2 expression and consequent decreased trastuzumab binding to HER2. We found that these trastuzumab-resistant cell lines showed phenotypic changes compared with parental cells, such as in their morphology and growth potential in vitro. However, detailed analysis clearly demonstrated that HER2 gene amplification, HER2 protein expression, and the binding of trastuzumab to HER2 remain essentially unchanged in the resistant cells, thereby excluding the first possibility. A second possibility is the overexpression of the p95HER2 fragments generated by the proteolytic cleavage of the extracellular domain or alternative initiation of translation from the AUG codon. In breast cancer, 20-40\% of HER2-positive tumors reportedly express p95HER2, and patients with p95HER2-positive tumors have a worse prognosis due to trastuzumab resistance [15]. Our two HER2-positive parental cell lines expressed p95HER2 with MWs ranging from 100 to $115 \mathrm{kDa}$, but no substantial increase in either total p95HER2 or phosphorylated p95HER2 was observed in the resistant cells, indicating no possibility of p95HER2 involvement in trastuzumab resistance. A third possibility is the overexpression of MUC4, a membrane-bound mucin glycoprotein, as reported for breast cancers. A transmembrane subunit of MUC4 that contains two EGF domains reportedly binds to the extracellular domain of HER2 and triggers specific phosphorylation of HER2, leading to the activation of downstream signaling pathways $[14,31]$. Alternatively, MUC4 reduces the binding of anti-ErbB2 antibodies to tumor cell surfaces by masking the extracellular domain of HER2 with high molecular weight sugar side chains. We found that MUC4 was upregulated at both the mRNA and protein levels in GLM-1HerR3 cells compared with parental GLM-1 cells. However, the binding of trastuzumab to the tumor cell surface was unchanged in trastuzumab-resistant cells. No apparent increase in the phosphorylation of HER2 or the consequent activation of downstream signaling pathways was observed in the resistant cells. In contast, MUC4 was downregulated in GLM-4HerR2 cells compared with parental GLM-4 cells. Therefore, the precise role of MUC4 in the trastuzumab resistance remains to be elucidated. Furthermore, protein expression of PTEN was not significantly reduced in trastuzumab-resistant cells compared with parental cells in vitro (data not shown). Further detailed studies, such as an in vivo analysis, are needed to clarify the mechanism of trastuzumab resistance in HER2-positive gastric cancer cell lines.

In conclusion, we developed new HER2 gene-amplified, trastuzumab-resistant gastric cancer cell lines and demonstrated the sensitivity of these trastuzumab-resistant cell lines to lapatinib for the first time. Although the precise mechanism of trastuzumab resistance is still unclear, these cell lines would be excellent preclinical models for understanding the mechanism of trastuzumab resistance and developing a new molecular targeting therapy (or for use in combination with chemotherapy) in patients with trastuzumab-resistant gastric cancers.

Acknowledgments The authors thank Ms. M. Yoshimura and N. Saito for expert technical assistance. This study was supported in part by a grant from the Ministry of Health, Labor and Welfare, Japan and Ministry of Education, Science, Sports, Culture and Technology, Japan.

\section{References}

1. Ferlay J, Shin HR, Bray F, Forman D, Mathers C, Parkin DM. Estimates of worldwide burden of cancer in 2008: GLOBOCAN 2008. Int J Cancer. 2010;127:2893-917.

2. Takehana T, Kunitomo K, Kono K, Kitahara F, Iizuka H, Matsumoto Y, et al. Status of c-erbB-2 in gastric adenocarcinoma: a comparative study of immunohistochemistry, fluorescence in situ hybridization and enzyme-linked immuno-sorbent assay. Int $\mathbf{J}$ Cancer. 2002;98:833-7.

3. Tanner M, Hollmen M, Junttila T, Kapanen A, Tommola S, Soini $\mathrm{Y}$, et al. Amplification of HER-2 in gastric carcinoma: 
association with Topoisomerase II $\alpha$ gene amplification, intestinal type, poor prognosis and sensitivity to trastuzumab. Ann Oncol. 2005; 16:273-8.

4. Yonemura Y, Ninomiya I, Yamaguchi A, Fushida S, Kimura H, Ohoyama S, et al. Evaluation of immunoreactivity for erbB-2 protein as a marker of poor short term prognosis in gastric cancer. Cancer Res. 1991;51:1034-8.

5. Yu GZ, Chen Y, Wang JJ. Overexpression of Grb2/HER2 signaling in Chinese gastric cancer: their relationship with clinicopathological parameters and prognostic significance. J Cancer Res Clin Oncol. 2009;135:1331-9.

6. Cobleigh MA, Vogel CL, Tripathy D, Robert NJ, Scholl S, Fehrenbacher L, et al. Multinational study of the efficacy and safety of humanized anti-HER2 monoclonal antibody in women who have HER2-overexpressing metastatic breast cancer that has progressed after chemotherapy for metastatic disease. J Clin Oncol. 1999;17:2639-48.

7. Slamon DJ, Leyland-Jones B, Shak S, Fuchs H, Paton V, Bajamonde A, et al. Use of chemotherapy plus a monoclonal antibody against HER2 for metastatic breast cancer that overexpresses HER2. N Engl J Med. 2001;344:783-92.

8. Smith I, Procter M, Gelber RD, Guillaume S, Feyereislova A, Dowsett M, et al. 2-year follow-up of trastuzumab after adjuvant chemotherapy in HER2-positive breast cancer: a randomised controlled trial. Lancet. 2007;369:29-36.

9. Fujimoto-Ouchi K, Sekiguchi F, Yasuno H, Moriya Y, Mori K, Tanaka Y. Antitumor activity of trastuzumab in combination with chemotherapy in human gastric cancer xenograft models. Cancer Chemother Pharmacol. 2007;59:795-805.

10. Bang YJ, Van Cutsem E, Feyereislova A, Chung HC, Shen L, Sawaki A, et al. Trastuzumab in combination with chemotherapy versus chemotherapy alone for treatment of HER2-positive advanced gastric or gastro-oesophageal junction cancer (ToGA): a phase 3, open-label, randomised controlled trial. Lancet. 2010;376:687-97.

11. Kute T, Lack CM, Willingham M, Bishwokama B, Williams H, Barrett K, et al. Development of Herceptin resistance in breast cancer cells. Cytometry Part A. 2004;57:86-93.

12. Lu Y, Zi X, Zhao Y, Mascarenhas D, Pollak M. Insulin-like growth factor-I receptor signaling and resistance to trastuzumab (Herceptin). J Natl Cancer Inst. 2001;93:1852-7.

13. Nagy P, Friedländer E, Tanner M, Kapanen AI, Carraway KL, Isola J, et al. Decreased accessibility and lack of activation of ErbB2 in JIMT-1, a herceptin-resistant, MUC4-expressing breast cancer cell line. Cancer Res. 2005;65:473-82.

14. Price-Schiavi SA, Jepson S, Li P, Arango M, Rudland PS, Yee L, et al. Rat Muc4 (sialomucin complex) reduces binding of antiErbB2 antibodies to tumor cell surfaces, a potential mechanism for herceptin resistance. Int J Cancer. 2002;99:783-91.

15. Scaltriti M, Rojo F, Ocana A, Anido J, Guzman M, Cortes J, et al. Expression of p95HER2, a truncated form of the HER2 receptor, and response to anti-HER2 therapies in breast cancer. J Natl Cancer Inst. 2007;99:628-38.

16. Park JG, Frucht H, LaRocca RV, Bliss DP Jr, Kurita Y, Chen TR, et al. Characteristics of cell lines established from human gastric carcinoma. Cancer Res. 1990;50:2773-80.

17. Kim SY, Kim HP, Kim YJ, Oh do Y, Im SA, Lee D, et al. Trastuzumab inhibits the growth of human gastric cancer cell lines with HER2 amplification synergistically with cisplatin. Int J Oncol. 2008;32:89-95.

18. Cho HS, Mason K, Ramyar KX, Stanley AM, Gabelli SB, Denney DW, et al. Structure of the extracellular region of HER2 alone and in complex with the Herceptin Fab. Nature. 2003;421:756-60.

19. Knuefermann C, Lu Y, Liu B, Jin W, Liang K, Wu L, et al. HER2/PI-3K/Akt activation leads to a multidrug resistance in human breast adenocarcinoma cells. Oncogene. 2003;22: 3205-12.

20. Li YM, Pan Y, Wei Y, Cheng X, Zhou BP, Tan M, et al. Upregulation of CXCR4 is essential for HER2-mediated tumor metastasis. Cancer Cell. 2004;6:459-69.

21. Geyer CE, Forster J, Lindquist D, Chan S, Romieu CG, Pienkowski T, et al. Lapatinib plus capecitabine for HER2-positive advanced breast cancer. N Engl J Med. 2006;355:2733-43.

22. Konecny GE, Pegram MD, Venkatesan N, Finn R, Yang G, Rahmeh M, et al. Activity of the dual kinase inhibitor lapatinib (GW572016) against HER-2-overexpressing and trastuzumabtreated breast cancer cells. Cancer Res. 2006;66:1630-9.

23. Kim JW, Kim HP, Im SA, Kang S, Hur HS, Yoon YK, et al. The growth inhibitory effect of lapatinib, a dual inhibitor of EGFR and HER2 tyrosine kinase, in gastric cancer cell lines. Cancer Lett. 2008;272:296-306.

24. Wainberg ZA, Anghel A, Desai AJ, Ayala R, Luo T, Safran B, et al. Lapatinib, a dual EGFR and HER2 kinase inhibitor, selectively inhibits HER2-amplified human gastric cancer cells and is synergistic with trastuzumab in vitro and in vivo. Clin Cancer Res. 2010;16:1509-19.

25. Nakanishi H, Yasui K, Ikehara Y, Yokoyama H, Munesue S, Kodera Y, et al. Establishment and characterization of three novel human gastric cancer cell lines with differentiated intestinal phenotype derived from liver metastasis. Clin Exp Metastasis. 2005;22:137-47.

26. Yokoyama H, Ikehara Y, Kodera Y, Ikehara S, Yatabe Y, Mochizuki $\mathrm{Y}$, et al. Molecular basis for sensitivity and acquired resistance to gefitinib in HER2-overexpressing human gastric cancer cell lines derived from liver metastasis. $\mathrm{Br} \mathrm{J}$ Cancer. 2006;95:1504-13.

27. Workman P, Balmain A, Hickman JA, McNally NJ, Rohas AM, Mitchison NA, et al. UKCCCR guidelines for the welfare of animals in experimental neoplasia. Lab Anim. 1988;22:195-201.

28. Nahta R, Takahashi T, Ueno NT, Hung MC, Esteva FJ. P27(kip1) down-regulation is associated with trastuzumab resistance in breast cancer cells. Cancer Res. 2004;64:3981-6.

29. Rowe DL, Ozbay T, Bender LM, Nahta R. Nordihydroguaiaretic acid, a cytotoxic insulin-like growth factor-I receptor/HER2 inhibitor in trastuzumab-resistant breast cancer. Mol Cancer Ther. 2008;7:1900-8.

30. Sawaki A, Ohashi Y, Omuro Y, Satoh T, Hamamoto Y, Boku N, et al. Efficacy of trastuzumab in Japanese patients with HER2positive advanced gastric or gastroesophageal junction cancer: a subgroup analysis of the Trastuzumab for Gastric Cancer (ToGA) study. Gastric Cancer. 2012;15:313-22.

31. Miyahara N, Shoda J, Ishige K, Kawamoto T, Ueda T, Taki R, et al. MUC4 interacts with ErbB2 in human gallbladder carcinoma: potential pathobiological implications. Eur J Cancer. 2008;44:1048-56. 